\title{
D0 Central Tracking Solenoid \\ Energization, Controls, Interlocks \& Quench Protection Operating Procedures
}

D0 Engineering Note 3823.111-EN- 526

August 26, 1998
ReV Jun 11, 200 !

R. Hance 


\section{Rick Hance \\ Engineering Note}

Date: 08/26/1998 Rev Date: 06/11/2001

Project: Solenoid Energization, Controls, Interlocks \& Quench Protection

Doc. No: H980826B (D0 Eng Note 3823.111-EN-526)

Subject: Solenoid Energization - Operating Procedures

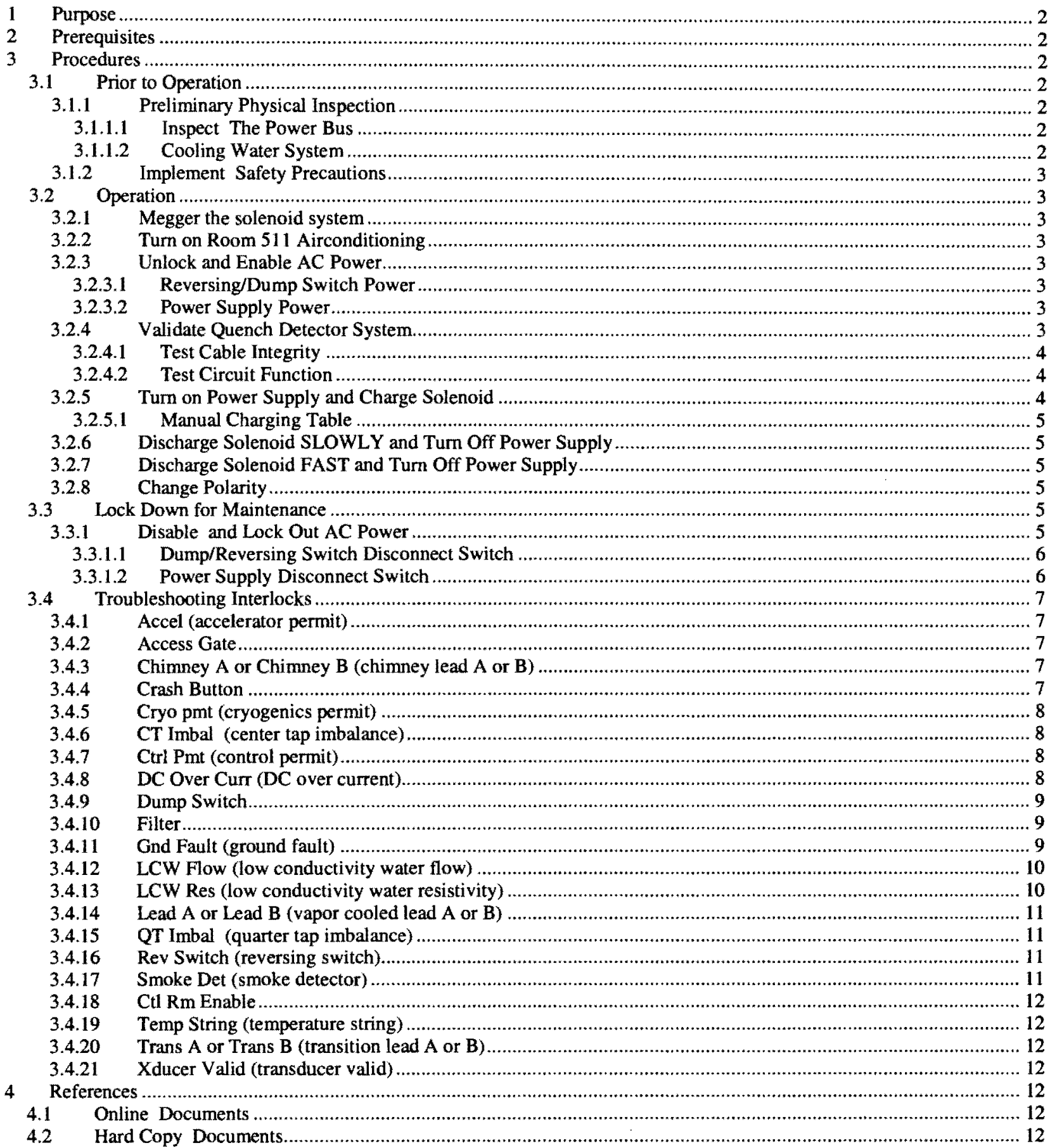




\section{Purpose}

This procedure is used when it is necessary to operate the solenoid energization, controls, interlocks and quench detection system. Note that a separate procedure exists for operating the solenoid "cryogenic" systems. Only D0 Control Room Operators or the Project Electrical Engineer are qualified to execute these procedures or operate the solenoid system.

\section{Prerequisites}

This procedure assumes that the operator is familiar with using the Distributed Manufacturing Automation and Control Software (DMACS). DMACS terminals are located in the Dzero Control Room, the Dzero Cryo Control Room, and several other locations around the site -- including some offices. Anyone logged in as a "PUBLIC" user may view the Solenoid Control Information on a DMACS terminal. However, In order to control the solenoid system, the user must have security privileges for "DO Sol DC". These privileges are assigned by the DMACS NT SER VER administrator (Dan Markley as of this writing). The operator should also have at least an understanding of the overall solenoid energization system as described in the references.

\section{Procedures}

\subsection{Prior to Operation}

\subsubsection{Preliminary Physical Inspection}

A preliminary inspection must be made to ensure that no obvious hazards to operation exist. This inspection is detailed in the following sub-sections.

\subsubsection{Inspect The Power Bus}

This step is necessary only if work has been done on or in the vicinity of the solenoid energization system since the system was previously operated. This is normally done by the Project Electrical Engineer or his designee at the end of an open or supervised access to the collision hall. Consult with the Project Electrical Engineer.

Verify the integrity of the DC bus beginning at the top of the control dewar located at the southeast corner of the detector. Continue the inspection all the way back to Room 511 on the south side of the detector building. Inspect all visible portions of the power bus. If any problems are found, then notify the Project Electrical Engineer before continuing. Examine the following items:

ㅁ The flexible cable connections that connect the power flags to the control platform water cooled bus. These connections shall be neatly insulated with silicon material and free of any extraneous test leads. The area around the connections shall be free of trash, abandoned tools and etc.

- The fixed building bus tray covers are all fastened in place.

․ The bus entering Room 511 is in fact attached to the solenoid energization system and all fasteners have paint seals to prove that they have been properly torqued. Note that there are two sets of buses and signal cables, one for the collision hall position and one for the assembly pit position of the solenoid. The correct buses must be connected as well as the correct signal cables. The connections are all labeled. Consult the Solenoid Project Electrical Engineer if in doubt.

- The solenoid dump resistor is in fact connected across the bus in Room 511. All fasteners must have paint seals to prove that they have been properly torqued.

\subsubsection{Cooling Water System}

Verify that the low conductivity water (LCW) cooling system is prepared to function by examining the following items.

- The four (4) manual LCW valves supplying equipment inside Room 511 are in the "ON" position.

- The LCW supply pressure gauge inside Room 511 reads at least 90 psi.

ㅁ There are no obvious water LCW leaks in Room 511 . 


\subsubsection{Implement Safety Precautions}

Skip this section for normal operation under secure conditions. If the solenoid is going to be operated when people may approach during operation, then ropes, signs, and flashing lights must be posted. Implement or verify the following precautions:

- Block the access to the control dewar ladder with yellow rope, flashing light, and "Caution, Magnet Energized, Authorized Personnel Only" sign.

a Block the access to the north end of the solenoid with yellow rope, flashing light, and "Caution, Magnet Energized, Authorized Personnel Only" sign.

a Block the access to the south end of the solenoid with yellow rope, flashing light, and "Caution, Magnet Energized, Authorized Personnel Only" sign.

- Make an announcement over the PA system at 30 minutes and 15 minutes to clear the solenoid area before the solenoid is about to be energized.

a Physically inspect the areas adjacent to the solenoid for personnel and loose equipment prior to energizing.

\subsection{Operation}

\subsubsection{Megger the solenoid system}

This step may be skipped if there has been no access to the collision hall or power supply room 511 since last operation i.e. if there is valid reason to believe the solenoid power circuit has not been disturbed. Consult with the Project Electrical Engineer.

1) Verify that the LCW Resistivity interlock is made up.

2) Unseat the power supply regulator module.

3) Place the "Ground Fault" switch in the "megger" position on the NIM ground fault module.

4) Place filter grounding knife switch into the "megger" position.

5) Connect the megger instrument to the megger test point and ground bus on the filter.

6) Verify that no personnel or equipment is in contact with the bus at any point.

7) Set the megger to $250 \mathrm{~V}$ and observe the reading. $20 \mathrm{k} \Omega$ or more @ $250 \mathrm{~V}$ is normal.

8) Remove megger, return grounding switch to normal position, replace power supply regulator module, and return the "Ground Fault" switch to the "Run" position on the NIM ground fault module.

\subsubsection{Turn on Room 511 Airconditioning}

Room 511 must be airconditioned to cool the solid bus work, dump resistor, dump switch, and reversing switch.

a Turn on the $\mathrm{CAC} 1$ and $\mathrm{CAC} 2$ disconnect switches on the west wall of room 511 and verify that the overhead airconditioners are working.

\subsubsection{Unlock and Enable AC Power}

The AC disconnect switches for the power supply and the reversing switch/dump switch cabinet are located on the west wall of Room 511. Each switch is clearly marked as to its function. When the solenoid is not operating, the switches are normally padlocked in the "OFF" position by configuration locks. These locks are managed by D0 Control Room Operators that have been trained and qualified by the Project Electrical Engineer or designee.

\subsubsection{Reversing/Dump Switch Power}

- Remove the padlock and engage the Reversing Switch/Dump Switch disconnect to the "ON" position. Leave the open padlock with the switch.

\subsubsection{Power Supply Power}

口 Remove the padlock and engage the Power Supply disconnect to the "ON" position. Leave the open padlock with the switch.

- Engage the Power Supply "Main" switch on the power supply front panel to the "ON" position.

\subsubsection{Validate Quench Detector System}

The quench detector circuitry is extremely fail safe by design. The most likely failure mode is for an override jumper to be left in the controls end rack during maintenance. The procedure described here should be exercised prior to operation if any solenoid system has received maintenance since the last operation. 


\subsubsection{Test Cable Integrity}

All quench detector cables are monitored by an active supervisory system. If any cable is opened or shorted or terminated in any way other than normal, then its associated quench detector interlock will not indicate "OK".

- Reset the system interlocks (DMACS interlock control screen).

- Verify that the following interlocks status are "OK" (interlock control screen):

- Lead A (vapor cooled lead A)

- Lead B (vapor cooled lead B)

- Trans A (transition lead A)

- Trans B (transition lead B)

- Chimney A (chimney lead A)

- Chimney B (chimney lead B)

- CT Imbal (center tap imbalance)

- QT Imbal (quarter tap imbalance)

\subsubsection{Test Circuit Function}

This test forces an artificial imbalance of the cable integrity circuits discussed above. NOTE: The dump switch will open during this test if it is closed. A FAST dump will occur during this test if the solenoid is operating .

口 Reset the system interlocks (DMACS interlock control screen). See section 3.4 if interlocks do not reset.

- Verify that the following interlocks status are "OK" (interlock control screen):

- Lead A (vapor cooled lead A)

- Lead B (vapor cooled lead B)

- Trans A (transition lead A)

- Trans B (transition lead B)

- Chimney A (chimney lead A)

- Chimney B (chimney lead B)

- CT Imbal (center tap imbalance)

- QT Imbal (quarter tap imbalance)

a Close the Dump Switch if it is not already closed (dump switch control screen).

a Trigger the quench detector test (select "Trigger" on the quench detector control screen).

- Verify that ALL of the interlocks status listed above are "Not OK" (interlock screen).

a Verify that the Dump Switch has opened (Dump Switch "Not Ok").

\subsubsection{Turn on Power Supply and Charge Solenoid}

Accomplish the following steps from the DMACs console. The operator must have security privileges of "D0 Sol DC" in order to execute any of these control functions. The interlock system is progressive and thus the steps must be executed in the order indicated. If any step is missed, just back up and proceed forward from the missed step.

- Verify that the power supply status is as follows (power supply screen):

Local/Remote Status $=$ Remote $>$

PS Regulation Mode $=$ Current $>$ If not correct, these conditions must be manually set at power supply PS Reference $=$ Ext $>$

- Enable the Controls Permit if necessary (interlock control screen)

口 Reset the interlocks (interlock control screen -- Note Dump switch won't reset until later)

- Close the Dump Switch (dump switch control screen)

ㅁ Reset the Power Supply (power supply control screen) -- Note: this also resets system interlocks including DS

- Verify that the Interlocks indicate "OK" (OK status should appear on interlock block) - see the

"Troubleshooting Interlocks" section if "Not OK"

- Set the Power Supply On (power supply control screen).

- Select "Use Auto VLIMIT>" (dynamically adjusts PS voltage for maximum safe charge rate).

- Set the Target Amps to desired current (power supply control screen). The normal target value is 4760 Amps to get to 2 Tesla. The system will reject any request $>4761$ Amps.

- Select "Proceed to Target" (power supply control screen). Uninterrupted charging requires $\cong 20$ minutes. The read back will indicate $\equiv 4748$ Amps in either polarity at 2 Tesla.

$\square$ Charging may be paused at any time by selecting "Hold at Present" (power supply control screen). Charging is resumed by entering a new Target Amps and selecting "Proceed to target".

h $980826 \mathrm{~b}$ - solenoid operating procedure.doc Page 4 of 12

(D0 Eng Note 3823.111.EN.526) 


\subsubsection{Manual Charging Table}

The solenoid can also be charged with manual instead of automatic control of the power supply voltage as follows:

- Set the Power Supply On (power supply control screen).

口 Select "Use Man. VLIMIT>" (power supply control screen).

- Set the manual VLIMIT to 3.0 volts (power supply control screen).

- Set the Target Amps to desired current (power supply control screen)

- Select "Proceed to Target" (power supply control screen)

Monitor the solenoid current during charging and periodically adjust the voltage limit as required. Do not exceed the limits listed on the following charge schedule. Reselect "Proceed to Target" after each new voltage is entered.

\begin{tabular}{|c|c|}
\hline - At $\mathrm{I}=0000 \mathrm{~A}$, set voltage limit to $3.0 \mathrm{~V}$ & - At $\mathrm{I}=2300 \mathrm{~A}$, set voltage limit to $5.5 \mathrm{~V}$ \\
\hline - At I $=0700 \mathrm{~A}$, set voltage limit to $3.5 \mathrm{~V}$ & - At I $=2800 \mathrm{~A}$, set voltage limit to $6.0 \mathrm{~V}$ \\
\hline At I $=1100 \mathrm{~A}$, set voltage limit to $4.0 \mathrm{~V}$ & - At I $=3400 \mathrm{~A}$, set voltage limit to $6.5 \mathrm{~V}$ \\
\hline - At $\mathrm{I}=1300 \mathrm{~A}$, set voltage limit to $4.5 \mathrm{~V}$ & - At I $=3900 \mathrm{~A}$, set voltage limit to $7.0 \mathrm{~V}$ \\
\hline - At $\mathrm{I}=1900 \mathrm{~A}$, set voltage limit to $5.0 \mathrm{~V}$ & - $\quad$ At I $=4300 \mathrm{~A}$, set voltage limit to $7.5 \mathrm{~V}$ \\
\hline
\end{tabular}

\subsubsection{Discharge Solenoid SLOWLY and Turn Off Power Supply}

a Set the Target Amps to 0 (DMACS power supply control screen).

- Set the Power Supply Off (DMACS power supply control screen) -- discharge will require $\approx 15$ minutes.

\subsubsection{Discharge Solenoid FAST and Turn Off Power Supply}

The quickest way to perform a FAST discharge is by opening the dump switch. This disables the power supply and forces the solenoid to discharge into the dump resistor rather than bypassing through the free wheeling diode. A FAST discharge stresses the system with resistive heating, mechanical forces, and high voltages. The stresses are well within design parameters. The quench detection system will trigger on the inductive voltages resulting from the rapid change of current with respect to time, and will require a reset after the discharge. The solenoid shell will absorb some energy in the form of heat. This may require some recovery time for the cryo system.

- Open the Dump Switch (DMACS dump switch control screen). .- interlocks will shutdown power supply, system will discharge in $\approx 40$ seconds.

\subsubsection{Change Polarity}

The system must be in a fully discharged state before it will reverse. Hardware and software interlocks provide protection from attempts to reverse the system while current is flowing. It will require $\approx 40$ minutes to discharge, change polarity, and recharge the solenoid.

a Set the Target Amps to 0 (enter Sol Target Amps $=0$ on power supply control screen).

口 Select "Proceed to Target" (select "Proceed to Target" on power supply control screen).

a Command the reversing switch to reverse (select "Proceed" on the reversing switch control screen). Note that nothing will happen until the current is $<25$ Amps -- at which time the reversing switch will change states.

D Wait for the Reversing Switch to Reverse as indicated on the console.

口 Follow the "Turn on Power Supply and Charge Solenoid" procedure to re-energize the solenoid.

\subsection{Lock Down for Maintenance}

The power supply and the dump/reversing switch cabinet should be locked off with operator's configuration padlocks when not in operation. In addition, anyone working on the solenoid energization system must lockout and tag out (LOTO) the device they are working on. There are no multiple energy source components in the system once it is discharged thus standard LOTO procedures apply. Follow the procedures below to apply configuration locks for routine lock down of the system.

\subsubsection{Disable and Lock Out AC Power}

The AC disconnect switches for the power supply and the reversing switch/dump switch cabinet are located on the west wall of Room 511 . Each disconnect switch is clearly marked as to its function. When the solenoid is not operating, the disconnect switches should be padlocked in the "OFF" position by configuration locks that are managed by the control room operators. 


\subsubsection{Dump/Reversing Switch Disconnect Switch}

This small disconnect switch supplies power to all circuits inside the dump/reversing switch cabinet.

- Verify that the solenoid is discharged (solenoid current $<25$ Amps).

- Move switch handle to the off position.

- Apply operator's configuration padlock.

\subsubsection{Power Supply Disconnect Switch}

This large disconnect switch supplies power to all circuits inside the PE150 power supply cabinet.

a Verify that the solenoid is discharged (solenoid current $<25 \mathrm{Amps}$ ).

- Move switch handle to the off position.

- Apply operator's configuration padlock. 


\subsection{Troubleshooting Interlocks}

The DMACS Interlocks Screen lists all the interlocks that can prevent the system from operating. The interlocks are further described below. If all interlocks fail to indicate "OK" after you do a "Reset" on the Interlocks Control Screen, then the system will not energize. Use this information to either remedy the problem or to make an informed consultation with the experts. Actual end rack assignments and cable numbers are detailed in the "as built" documentation available on the www, or in hardcopy. See the references in section 4 at the end of this document.

\subsubsection{Accel (accelerator permit)}

This circuit consists of a relay contact controlled by the Accelerator Interlock System. It allows the solenoid to operate if the main ring door interlock system is intact, no keys are missing from the key tree, and the key tree door is closed. The contact is located in box titled "D0 Collision Hall \#96 Power Supply Interlock Unit". This box is located in rack F10923 located in the Dzero Control Room. The Beams Division personnel responsible for accelerator interlocks are Howard Casebolt and John E. Anderson. If this interlock can not be reset, then check the following:

- Main Ring Interlocks (call Main Control Room for status)

- D0 Collision Hall \#96 Power Supply Interlock Unit described above (call John E. Anderson or Howard Casebolt)

- Cable from "D0 Collision Hall \#96 Power Supply Interlock Unit" to end rack in room 511

- NIM Interlock module in slot 8 of NIM crate and cabling

- PLC Input module in slot 11 of PLC controls crate and cabling

\subsubsection{Access Gate}

This interlock is controlled by a switch on the access gate into the fenced-in area of room 511. This interlock is "Not Ok" if the gate is open. If this interlock will not reset, then check the following:

- Access gate -- must be closed

- Gate switch and cabling

- NIM interlock module in slot 9 of NIM crate and cabling (receives access gate permit)

- PLC input module in slot 12 of the PLC crate and cabling (monitors access gate interlock)

\subsubsection{Chimney A or Chimney B (chimney lead A or B)}

These circuits monitor for a voltage drop across chimney leads A or B. These interlocks should only read "Not Ok" after a quench of chimney lead A or B; or after a test of the quench system as described previously. If this interlock will not reset, then check the following:

- NIM power supply in controls rack in room 511. Note: spare power supply is in rack.

- Cable from voltage tap connectors on solenoid north end to resistor \& test point box near control dewar

- Resistor \& test point box at control dewar

- Cable from resistor \& test point box to voltage tap junction box in room 511

- Cable from the junction box in room 511 to the controls end rack

- NIM Amplifier module in NIM slot 4 and cabling

- NIM Interlock module in NIM slot 7 and cabling

- PLC Input module in PLC controls crate slot 11 and cabling

\subsubsection{Crash Button}

This circuit consists of two crash buttons in series. If this interlock can not be reset, then check the following:

- "Toroid/Solenoid Enable" button on control room "Power Disablement Panel"

- Crash button in collision hall labyrinth

- Cable from "Toroid/Solenoid Enable" button to controls end rack in room 511 via cable cross connect in SW corner of control room

- Cable from crash button in collision hall labyrinth to controls end rack in room 511 via cable cross connect in SW corner of control room

- NIM interlock module in slot 8 of NIM crate and cabling

- PLC Input module in slot 12 of PLC controls crate and cabling 


\subsubsection{Cryo pmt (cryogenics permit)}

This interlock is controlled by a relay contact in a PLC crate in room 510 (cryogenics PLC crate). This interlock is "Not Ok" if the PLC determines several important cryogenic system parameters to be out of limits. If this interlock will not reset, then check the following:

- NIM interlock module in slot 9 of NIM crate and cabling (receives cryo permit)

- PLC input module in slot 12 of the PLC crate and cabling (monitors cryo interlock)

- Call cryo system expert (Dan Markley)

\subsubsection{CT Imbal (center tap imbalance)}

This circuit monitors for an imbalance between the actual solenoid center tap voltage, and the voltage at the junction of a voltage divider across the solenoid. The voltage divider provides $1 / 2$ of the total solenoid voltage at the junction. This interlock should only read "Not Ok" after a quench of one or more solenoid coils, or after a test of the quench system as described previously. If this interlock will not reset, then check the following:

- NIM power supply in controls rack in room 511. Note: spare power supply is in rack.

- Cable from voltage tap connectors on solenoid north end to resistor \& test point box near control dewar

- Resistor \& test point box at control dewar

- Cable from resistor \& test point box to voltage tap junction box in room 511

- Cable from the junction box in room 511 to the controls end rack

- $\quad$ NIM Bridge module in NIM slot 1 and cabling

- NIM amplifier module in NIM slot 6 and cabling

- NIM Interlock module in NIM slot 7 and cabling

- PLC Input module in PLC controls crate slot 11 and cabling

\subsubsection{Ctrl Pmt (control permit)}

This circuit is a relay contact in the programmable logic controller (PLC) controls crate which is controlled by DMACS software. The Operator can turn it "ON" or "OFF" in the "Interlock Control" screen. Also, ANYONE can turn it "OFF" by selecting "Emergency Shutoff" from the "Main" screen. This interlock WILL NOT reset automatically. The Operator must "Set Cntrl Pmt" ON manually in the "Interlock Control" screen before resetting interlocks. If this interlock can not be turned $O N$ in the "Interlock Control" screen, then check the following:

- PLC Relay Output Module in slot 9 of the PLC controls crate and cabling

- Interlock Module in slot 8 of NIM crate and cabling

- PLC Input module in slot 11 of PLC controls crate and cabling

\subsubsection{Over Curr (DC over current)}

This interlock is controlled by the NIM "Absolute Value " module in the NIM crate in room 511. The interlock is "Not Ok" if the module determines that either the solenoid current or the power supply current is in excess of a preset value $>5000$ Amps. It signifies that the current has exceeded its design limits. If this interlock fails to reset, then check the following:

- NIM power supply in controls rack in room 511. Note: spare power supply is in rack.

- NIM absolute value module in NIM crate and cabling

- NIM interlock module in slot 9 of NIM crate and cabling (receives dc overcurr permit)

- PLC input module in slot 12 of the PLC crate and cabling (monitors dc overcurr interlock)

- Holec transductor chassis and cables in controls rack 


\subsubsection{Dump Switch}

This interlock is controlled by an auxiliary relay contact on the 5000 Amp dump switch. The interlock is "Not Ok" when the dump switch is open, and "Ok" when the dump switch is closed. If this interlock fails to reset, then check the following:

- All eight "quench detector" interrupts must be "Ok" before dump switch can be closed (Lead A, Lead B, Trans A, Trans B, Chimney A, Chimney B, CT imbalance, and QT Imbalance)

- Close Dump Switch (Dump Switch control screen)

- NIM amplifier modules in slot 2,3,4, and 6 of NIM crate and cabling (opens dump switch)

- NIM interlock module in slot 7 of NIM crate and cabling (receives QD permits and opens dump switch redundantly)

- NIM interlock module in slot 9 of NIM crate and cabling (receives dump switch permit)

- PLC input module in slot 12 of the PLC crate and cabling (monitors interlock)

- PLC relay output module in slots 9 and 10 of PLC crate (opens and resets dump switch)

\subsubsection{Filter}

This circuit consists of the contacts of the hipot/normal switch in series with pressure safety switches on two large "can" capacitors mounted on the filter assembly. The interlock is "Ok" if the hipot switch is in the "normal" position, and the capacitors have not vented and opened their pressure switches. If this interlock fails to reset, then check the following:

- Position of Filter hipot switch (large knife-blade switch located on filter assembly -- must be in Run position)

- Pressure switches on large metal "damping resistor blocking capacitors" on filter assembly

- NIM interlock module in slot 8 of the NIM crate and cabling (receives filter permit)

- PLC input module in slot 12 of the PLC crate and cabling (monitors interlock)

\subsubsection{Gnd Fault (ground fault)}

This interlock is controlled by the NIM "Ground Fault Detector " module in the NIM crate in room 511. The interlock is "Not Ok" if the NIM module fails or if the resistance to ground from the center tap of the Dump Resistor is < a preset low limit or > a preset upper limit. If this interlock fails to reset, then check the following:

- Position of Filter hipot switch (large knife-blade switch located on filter assembly -- must be in Run position)

- NIM Ground Fault Detector module in NIM crate and cabling (monitors gnd resistance and provides ground fault permit)

- NIM interlock module in slot 9 of NIM crate and cabling (receives ground fault permit)

- PLC input module in slot 12 of the PLC crate and cabling (monitors ground fault interlock) 


\subsubsection{LCW Flow (low conductivity water flow)}

This circuit consists of a relay contact controlled by the solenoid PLC in response to conditions in two systems. The two systems include: 1) The PS and Filter LCW system, and 2) The bus and diode LCW system. The conditions include: power supply current, transformer temperature, and power supply LCW flow switch status; and bus current, free wheeling diode temperature, and bus LCW flow switch respectively. To prevent condensation, the respective LCW valves are not opened until the solenoid current is $>50 \mathrm{Amps}$ or temperatures are $>40^{\circ} \mathrm{C}$. Then, to provide hysteresis, the valves remain open until the solenoid current is $<25$ Amps and temperatures are $<35^{\circ} \mathrm{C}$.

The interlock will indicate "Ok" as long as the solenoid current is $<100 \mathrm{Amps}$ and temperatures are $<45^{\circ} \mathrm{C}$; or the flow switches are indicating that LCW is flowing. There are two failure scenarios to be considered:

- Interlock fails to reset before charging the solenoid. Check the following and notify expert of any unexplainable discrepancy:

- PS current reading $<25 \mathrm{~A}$

- Bus current reading $<25 \mathrm{~A}$

- Interphase Xfmr temp $<35^{\circ} \mathrm{C}$

- Free wheel diode temp $<35^{\circ} \mathrm{C}$

- PS \& Filter LCW Sol Valve indicates "Off"

- Sol Bus and Diode LCW Sol Valve indicates "Off"

- PS \& Filter LCW Flow Switch indicates "No Flow"

- Sol Bus and Diode LCW Flow Switch indicates "No Flow"

- LCW Flow Status to Interlocks indicates "Ok" -- If not, check PLC relay output module in slot 10 of PLC crate and cabling (controls flow status signal); and NIM interlock module in slot 8 of NIM crate and cabling (monitors flow status signal)

口 Interlock trips shortly after starting a charging cycle. Before proceeding, check the following:

- LCW pumps are operating (60 psi pressure differential on LCW gauges in room 511)

- The four manual LCW valves in room 511 are "On".

Then, reset the interlocks and charge the solenoid to 75 Amps. Keeping the current at 75 amps will signal the PLC to energize the LCW valves; but will be less than the 100 Amps that will cause an interlock trip if the flow switches do not indicate that LCW is flowing. Monitor the LCW screen, check the following, and notify expert of any unexplainable discrepancy:

- PS current reading $\approx 75 \mathrm{~A}$

- Bus current reading $\approx 75 \mathrm{~A}$

- PS \& Filter LCW Sol Valve indicates "On"

- Sol Bus and Diode LCW Sol Valve indicates "On"

- PS \& Filter LCW Flow Switch indicates "Flow"

- Sol Bus and Diode LCW Flow Switch indicates "Flow"

- LCW Flow Status to Interlocks indicates "Ok" -- If not, check PLC relay output module in slot 10 of PLC crate and cabling (controls flow status signal); and NIM interlock module in slot 8 of NIM crate and cabling (monitors flow status signal)

\subsubsection{LCW Res (low conductivity water resistivity)}

This circuit consists of a relay contact in a "Beckman Industrial Solu Meter" located in the low conductivity water (LCW) supply in DAB 6th floor west mechanical room. The meter measures the conductivity of the LCW that is used to cool the power supply, filter, and water cooled bus. If this interlock can not be reset, then check the following;

- Specific resistance of the LCW is below the Beckman meter set point (set point should be 4.0 MegOhms$\mathrm{cm})$

- Beckman meter

- Cable from Beckman meter to controls end rack in room 511

- NIM Interlock module in slot 8 of NIM crate and cabling

- PLC Input module in slot 12 of PLC controls crate and cabling 


\subsubsection{Lead A or Lead B (vapor cooled lead A or B)}

These circuits monitor for a voltage drop across vapor cooled lead A and B. These interlocks should only read "Not $\mathrm{Ok}^{\prime}$ after the voltage drop across lead A or B has exceeded a threshold due to excessive temperature of the leads; or after a test of the quench system as described previously. If this interlock will not reset, then check the following:

- NIM power supply in controls rack in room 511. Note: spare power supply is in rack.

- Cable from voltage tap connectors on control dewar to resistor \& test point box

- Resistor \& test point box at control dewar

- Cable from resistor \& test point box to voltage tap junction box in room 511

- Cable from the junction box in room 511 to the controls end rack

- NIM Amplifier module in slot 2 of the NIM crate and cabling

- NIM Interlock module in slot 7 of the NIM crate and cabling

- PLC Input module in PLC controls crate slot 11 and cabling

\subsubsection{QT Imbal (quarter tap imbalance)}

This circuit monitors for an imbalance between the actual solenoid quarter tap voltage, and the voltage at the junction of a voltage divider across the solenoid. The voltage divider provides $1 / 4$ of the total solenoid voltage at the junction. This interlock should only read "Not Ok" after a quench of the solenoid, or after a test of the quench system as described previously. If this interlock will not reset, then check the following:

- NIM power supply in controls rack in room 511. Note: spare power supply is in rack.

- Cable from voltage tap connectors on solenoid north end to resistor \& test point box near control dewar

- Resistor \& test point box at control dewar

- Cable from resistor \& test point box to voltage tap junction box in room 511

- Cable from the junction box in room 511 to the controls end rack

- NIM Bridge module in NIM slot 1 and cabling

- NIM amplifier module in NIM slot 6 and cabling

- NIM Interlock module in NIM slot 7 and cabling

- PLC Input module in slot 11 of PLC controls crate and cabling

\subsubsection{Rev Switch (reversing switch)}

This interlock is controlled by an auxiliary relay contact on the power supply reversing switch. The interlock is "Not OK" if the reversing switch is "cycling" (switching motor is running). If this interlock fails to reset, then check the following:

- Reversing switch is cycling (wait 1 minute to complete)

- NIM interlock module in slot 9 of NIM crate and cabling (receives reversing switch permit)

- PLC input module in slot 12 of the PLC crate and cabling (monitors reversing switch interlock)

\subsubsection{Smoke Det (smoke detector)}

This interlock is controlled by the "rack monitor interface" chassis (RMI) in the top of the controls rack. This interlock is "Not Ok" if the smoke detector in the top of the rack has sensed smoke in the rack. If this interlock will not reset then check the following:

- RMI Chassis in top of controls rack in room 511 -- Manually reset the chassis -- WARNING! If the smoke detector LED on the chassis is lit, then smoke has probably been sensed. Even if the smoke is not present now, the cause of the trip must be determined before continuing.

- Smoke detector Head

- NIM interlock module in slot 9 of NIM crate and cabling (receives smoke detector permit)

- PLC input module in slot 12 of the PLC crate and cabling (monitors smoke detector interlock) 


\subsubsection{CtI Rm Enable}

This circuit is connected to the Solenoid/Toroid Enable switch in the control room "Safety Panel". Verify that this switch is in the "enable " position. Notify the Project Electrical Engineer if this interlock will not reset.

\subsubsection{Temp String (temperature string)}

This circuit consists of a string of $\mathbf{4 0}$ Klixon bi-metallic temperature sensitive switches strategically located at various points throughout the solenoid energization system. If this interlock fails to reset, then check the following:

- NIM interlock module in slot 8 of the NIM crate and cabling (receives temp permit)

- PLC input module in slot 12 of the PLC crate and cabling (monitors interlock)

- Control dewar Klixons (requires expert \& sheet 15 of schematics)

- Room 511 Klixons (requires expert \& sheet 15 of schematics)

\subsubsection{Trans A or Trans B (transition lead A or B)}

These circuits monitor for a voltage drop across transition lead A or B. These interlocks should only read "Not Ok" after a quench of transition leads $A$ or $B$; or after a test of the quench system as described previously. If this interlock will not reset, then check the following:

- NIM power supply in controls rack in room 511. Note: spare power supply is in rack.

- Cable from voltage tap connectors on control dewar to resistor \& test point box

- Resistor \& test point box at control dewar

- Cable from resistor \& test point box to voltage tap junction box in room 511

- Cable from the junction box in room 511 the controls end rack

- NIM Amplifier module in NIM slot 3 and cabling

- NIM Interlock module in NIM slot 7 and cabling

- PLC Input module PLC controls crate slot 11 and cabling

\subsubsection{Xducer Valid (transducer valid)}

This interlock is controlled by the NIM "Absolute Value " module in the NIM crate in room 511. The interlock is "Not Ok" if the module determines that the solenoid current reading from the Holec transductor is more that 25 Amps less than expected by comparing it with the power supply transductor reading minus the dump resistor current. It signifies that one of the current transducers is invalid. If this interlock fails to reset, then check the following:

- NIM power supply in controls rack in room 511. Note: spare power supply is in rack.

- NIM absolute value module in NIM crate

- NIM interlock module in slot 9 of NIM crate and cabling (receives xducer valid permit)

- PLC input module in slot 12 of the PLC crate and cabling (monitors xducer valid interlock)

- Holec transductor chassis and cables in controls rack

\section{References}

\subsection{Online Documents}

Detailed specifications, schematics, wiring assignments, component design reports and etc. can be viewed on-line with a world wide web (www) browser. The www address is as follows:

\section{http://www-d0.fnal.gov/-hance/solenoid.htm}

\subsection{Hard Copy Documents}

"As built" maintenance documents, including detailed specifications, schematics and wiring assignments are kept available and up-to-date in the power supply room (room 511); and also in the office of the system engineer (Rick Hance).

Full schematics are available for copying in the Dzero flat files in addition to the www. The schematics are fully hierarchical and are comprised of a set of 17 D-size drawings. The drawing number is 3823-111-ED-330052.

The detailed specification and description of the system is available for copying in the Dzero Engineering Notes system in addition to the www. The Engineering Note is 3823-111-EN-418. 